\title{
Un campo científico en busca de visibilidad. Jornadas internacionales sobre la calidad de las revistas de Ciencias de la Actividad Física y el Deporte
}

\author{
Por José Devís-Devís y Miguel Villamón-Herrera
}

\begin{abstract}
Resumen: El campo de las Ciencias de la Actividad Física y el Deporte (CCAFD) en España necesita de actuaciones que contribuyan a mejorar la calidad de sus publicaciones periódicas. De esta manera las revistas pueden convertirse en órganos eficientes de comunicación y difusión del conocimiento científico creado por sus investigadores. En este trabajo se detalla la realización de las "Jornadas internacionales sobre la calidad de las revistas de ciencias de la actividad física y el deporte" celebradas en Valencia el 25 y 26 de octubre de 2007. Durante esos días se discutió sobre distintas visiones de la calidad de las revistas, ejemplos de gestión editorial y estrategias concretas de mejora. Con estas Jornadas se ha pretendido contribuir a la visibilidad internacional del joven campo español de las CCAFD.
\end{abstract}

Palabras clave: Revistas científicas, Calidad, Ciencias de la actividad física y el deporte, Visibilidad, Comunicación científica.

Title: A scientific field searching visibility. International Workshop on Physical Activity and Sport Sciences Quality Journals

Abstract: The field of Physical Activity and Sport Sciences (PASS) in Spain requires activities that contribute to the improvement of its journals quality. Thus, journals may become efficient ways of diffusion and communication of scientific knowledge developed by their researchers. The present paper describes the development of the "International Workshop on Physical Activity and Sport Sciences Quality Journals" held in Valencia on the $25^{\text {th }}$ and $26^{\text {th }}$ of October 2007. Different views on journals quality, editorial process examples and specific strategies of improvement were discussed during these days. This Workshop represents a step further in the contribution to the international visibility of the young Spanish field of PASS.

Keywords: Scientific journals, Quality, Physical activity and sport sciences, Visibility, Scientific communication

Devís-Devís, José; Villamón-Herrera, Miguel. "Un campo científico en busca de visibilidad. Jornadas internacionales sobre la calidad de las revistas de Ciencias de la Actividad Física y el Deporte”. En: El profesional de la información, 2008, marzo-abril, v. 17, n. 2, pp.242-246.

DOI: $10.3145 /$ epi.2008.mar.18

\section{Introducción}

Los días 25 y 26 de octubre de 2007 se celebraron en Valencia las Jornadas internacionales sobre la calidad de las revistas de ciencias de la actividad física y el deporte $(C C A F D)$. En el Salón de Grados de la Facultad de CCAFD de la Universidad de Valencia se dieron cita más de cincuenta participantes, entre directores y representantes de los consejos editoriales de varias revistas, investigadores y bibliotecarios, e interesados en la temática, además de los once ponentes españoles y extranjeros.

La inauguración oficial de las Jornadas corrió a cargo del Vicerrector de la Universidad organizadora, el profesor Antonio Ariño, quien se refirió al grado de madurez alcanzado por la Licenciatura en CCAFD desde su integración universitaria. En su intervención también resaltó el importante papel que adquiere la calidad de las revistas dentro del Espacio Europeo común para la Enseñanza Superior y la Investigación. Tal y como se comentó desde la mesa, la organización de 
estas Jornadas surgió de la necesidad de aumentar la visibilidad de la producción española de este campo de la ciencia y contribuir a que los resultados de sus investigaciones lleguen a influir en el tejido productivo, en la solución de problemas sociales y en la mejora del bienestar de los ciudadanos y su medio ambiente. Para conseguirlo resulta fundamental la comunicación y la difusión de los resultados de las investigaciones, uno de los aspectos que pretende reforzar la Estrategia de Lisboa en cada uno de los países europeos y las distintas ramas de la ciencia.

A pesar de ello en España se observa una falta de rentabilidad en la inversión realizada en $\mathrm{I}+\mathrm{D}$, de manera más acusada en un campo poco consolidado como el de las CCAFD. Entre otras razones esta situación se debe a la existencia de pocas revistas españolas de prestigio que ofrezcan las garantías de calidad a la comunidad científica para una efectiva comunicación y diseminación de los resultados derivados de sus investigaciones. Por ello resulta trascendental que todos los agentes implicados en el ciclo de la ciencia contribuyan a aumentar el nivel de la calidad de las revistas españolas del campo de las CCAFD. Precisamente estas Jornadas constituyen una actuación modesta, pero importante, en el proceso por conseguir dicho propósito.

\section{El contenido}

El programa científico estuvo estructurado en torno a ocho conferencias, seguidas de turno de preguntas y debate, y una mesa redonda. Se pretendía con este programa ofrecer distintas visiones sobre la calidad de las revistas, ejemplos de gestión editorial y estrategias concretas de mejora. Para ello se contó con las aportaciones de los editores de revistas europeas de prestigio del campo mencionado, editores de revistas españolas de otros cam-

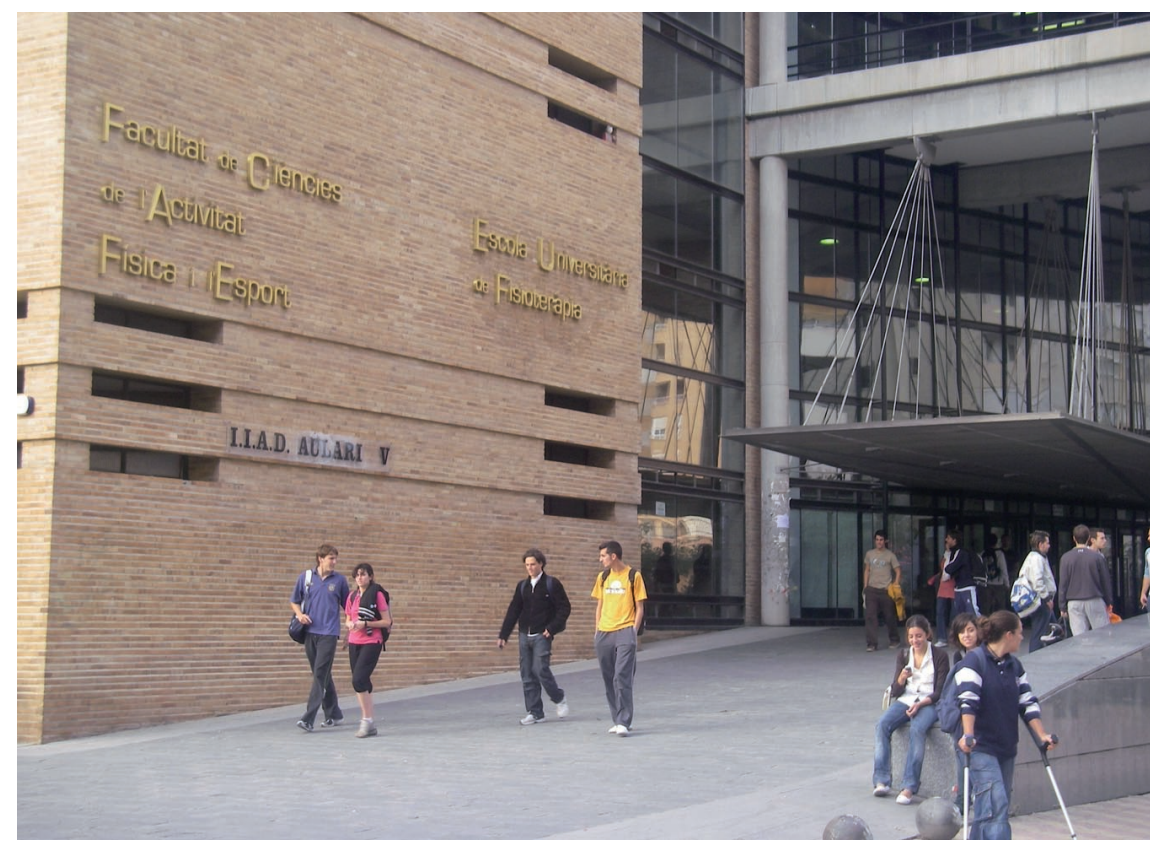

pos, investigadores e investigadoras especializadas en la evaluación de revistas de Medicina y Ciencias Sociales, así como un representante de la Fundación Española para la Ciencia y la Tecnología (Fecyt).

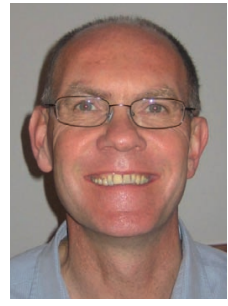

David Kirk
Las conferencias se iniciaron con David Kirk, editor de la revista Physical Education and Sport Pedagogy. Este ponente se refirió al factor de impacto (FI), el índice- $h$, el índice$g$ y los análisis que aporta Google scholar como formas de entender la calidad basadas en el análisis de citas. También ofreció otras alternativas para juzgar la calidad basadas en los análisis electrónicos de acceso a las revistas como son el listado de las descargas realizadas por los lectores durante un periodo dado, el listado de los artículos más leídos o el de los artículos más citados de entre los publicados por las propias revistas. En relación con la popularidad del FI, afirmó que "las revistas no deben permitir que un organismo totalmente privado como Thomson Scientific determine la medida de la calidad de las publicaciones periódicas". Además, señaló que no puede definirse la calidad únicamente a partir del FI y que debemos recurrir a múltiples fuentes de análisis o indicadores para emitir juicios sobre la calidad de las revistas científicas.

David Brown, profesor de la Universidad de Exeter (Reino Unido), profundizó en el tema de la calidad desde un punto de vista cualitativo. Para ello propuso las ideas de 'impacto subjetivo' e 'impacto

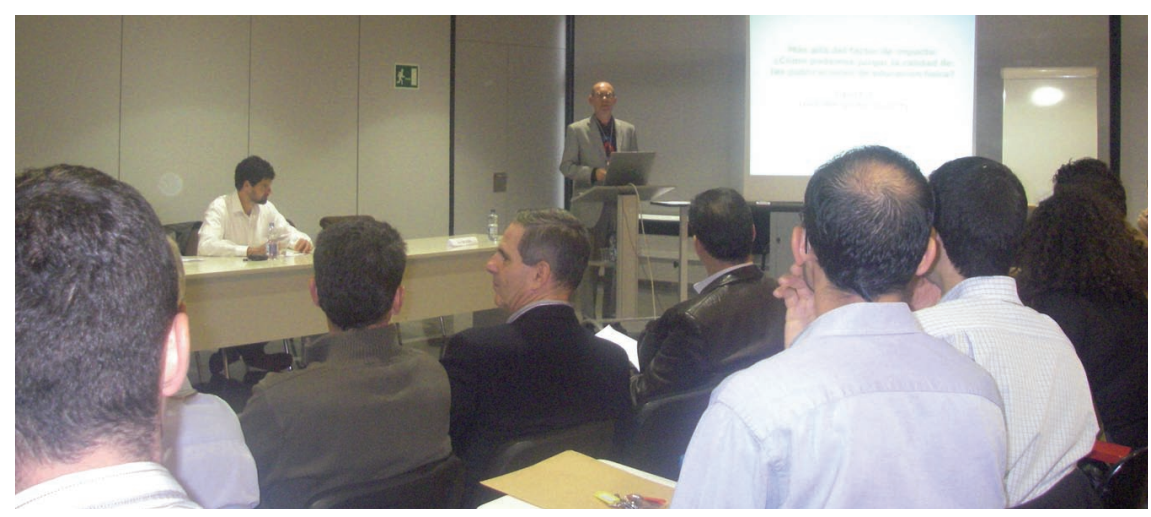


práctico’ que amplían la discusión del impacto a la influencia que un artículo tiene en las emociones y creencias más profundas de la gente o en las prácticas sociales diarias. Respecto al análisis de citas, señaló que no todas son iguales por lo que habría que distinguir entre las que indican la calidad de un artículo y las que no tienen que ver con este concepto, tales como las indiscriminadas o de relleno (bumper sticker) y las negativas. Por último, se refirió al anglocentrismo de las publicaciones con FI, ya que refuerza el imperialismo cultural, algo que considera muy negativo para las Ciencias Sociales. En su opinión, en las CCAFD se necesitan revistas plurilingües de alta calidad (con resúmenes en inglés y artículos en la lengua más apropiada para cada autor o autora y temática) y que sean de acceso libre en Internet.

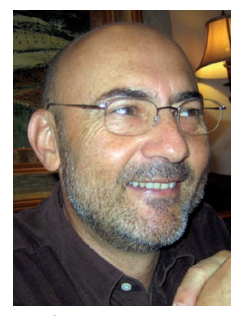

Rafael Aleixandre
La conferencia de Rafael Aleixandre, científico del CSIC-Universidad de Valencia, tuvo una orientación más práctica, ya que se dirigió a ofrecer estrategias de mejora para las revistas de CCAFD, a partir de las deficiencias detectadas en las revistas de este campo y de la Medicina. En el transcurso de su intervención realizó un exhaustivo repaso de todos los aspectos que conforman la calidad de las revistas científicas, en su vertiente formal, de contenido científico y difusión. También explicó las diversas tentativas que se vienen desarrollando para la elaboración de un FI de las revistas españolas por diversos grupos de investigación tales como el EC3 de la Universidad de Granada, el del Cindoc y el del Instituto de Historia de la Ciencia y Documentación López Piñero de Valencia. Concluyó su participación recomendando diversas estrategias puntuales como el estricto cumplimiento de los estándares internacionales de normalización y la elaboración de resúmenes de calidad (a ser posible estructurados) con su correcta traducción al inglés porque muchos investigadores no llegan a leer el artículo completo. Por supuesto, también mencionó la revisión exigente de los manuscritos por parte de los expertos, el aumento de la difusión mediante ediciones bilingües y el acceso libre en Internet.

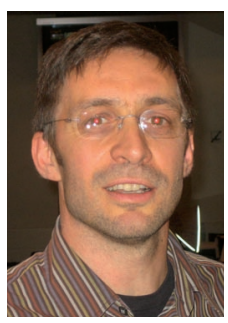

Las estrategias electrónicas para la mejora de la difusión de las revistas fueron el centro de la aportación realizada por Ernest Ernest Abadal Abadal, profesor de Biblioteconomía y Documentación en la Universidad de Barcelona. En particular, recomendó el paso a formato digital para ganar en visibilidad, la creación de portales de revistas y su inclusión en los existentes, la utilización de gestores de contenido, la difusión en libre acceso, la internacionalización de las revistas y la profesionalización de los equipos editoriales. Posteriormente, analizó la relación entre el formato digital y el acceso libre (open access) con la calidad, destacando la idea de que la calidad está relacionada con el cumplimiento de un conjunto de indicadores (formales, de contenido y difusión) y no con el formato (digital) o la forma de difusión.

Precisamente la baja presencia de las revistas españolas en las más prestigiosas bases de datos internacionales, como es la Web of Knowledge de Thomson Scientific, llevó a la Fecyt a lanzar el programa de Ayuda a las revistas científicas españolas (ARCE).

Así lo comentó José-Manuel Báez, representante de esta institución en las Jornadas, que también explicó las principales actuaciones en materia de profesionalización e internacionalización de las revistas españolas. Entre dichas actuaciones

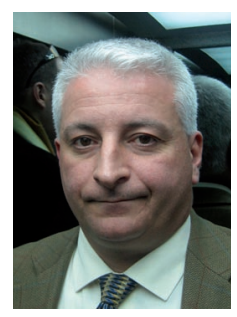
destaca la elaboración de un manual de edición para las revistas, la creación del Repositorio Español de Ciencia y Tecnología (Recyt) y una Red de repositorios institucionales para aumentar la visibilidad de la ciencia española, así como la cesión a las revistas que se integren en Recyt de OJS, una herramienta de gestión integral que les permita adaptarse a la publicación electrónica. Finalmente, invitó a los editores del campo de las CCAFD a que participaran en la recién estrenada convocatoria de ayuda a las revistas científicas.

\section{http://recyt.fecyt.es}

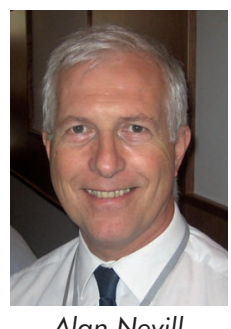

Alan Nevill, editor jefe de la revista Journal of sports sciences, recordó que el primer objetivo para cualquier investigador $\mathrm{o}$ investigadora consiste en publicar en las revistas de buena calidad. Desde su punto de vista, la calidad de una revista depende del índice de citación, el FI, la circulación, el índice de aceptación y la presencia en bases de datos de prestigio. Señaló que el FI en exclusividad no garantiza la calidad de una revista y que, a veces, se equivocan en su cálculo, tal y como ocurrió con la revista que dirige. Para este profesor de la Universidad de Wolverhampton (Reino Unido), la calidad de una revista descansa, en última instancia, en el rigor metodológico y en el trabajo del editor o editora y los revisores. Explicó que antes de aceptar los originales para publicar analizan los métodos de trabajo utilizados por los autores, y en concreto, cuando viene al caso, ponen como condición que se hayan hecho los ensayos clínicos con control doble-ciego aleatorio (individuos con medicamento e individuos con placebo). También ex- 
plicó el trabajo de los editores que deben hacer pedagogía entre sus lectores y autores potenciales.

En las jornadas también se presentaron dos modelos de revistas españolas que pudieran ser de interés para la audiencia. Una de ellas es El profesional de la información $(E P I)$, la primera revista del campo de la documentación que se ha integrado en el Social Science Citation Index. Su director, Tomás Baiget, hizo un poco de historia de la revista y enfatizó la importancia de presentarla en formato electrónico para aumentar su difusión y cuidar un diseño y calidad de impresión en el formato papel. La otra revista, Psicológica, también incluida recientemente en las bases de datos de Thomson Scientific, fue presentada por su director Salvador Algarabel como una revista exclusivamente electrónica de bajo coste. Este profesor de la Universidad de Valencia alabó las actuaciones recientes de la Fecyt en su apoyo a las revistas españolas, pero reclamó mayor ayuda para aumentar la profesionalización del proceso editorial de muchas revistas como la suya, que se sustentan con grandes dosis de altruismo y generosidad de sus responsables.

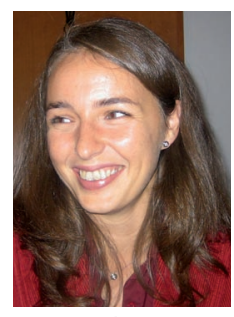

Elea Giménez-Toledo
Elea Giménez, investigadora del Cindoc y especialista en evaluación de revistas de Ciencias Sociales y Humanas, presentó los dos productos que se han elabora-

do desde el grupo de investigación del que forma parte. Se trata de la base de datos Difusión y calidad editorial de las revistas españolas de humanidades y ciencias sociales y jurídicas (DICE) que tiene como objetivo facilitar el conocimiento y la consulta de algunas de las características editoriales de las revistas españolas de Humanidades y Ciencias Sociales más estrechamente ligadas a la calidad. La Aneca utiliza

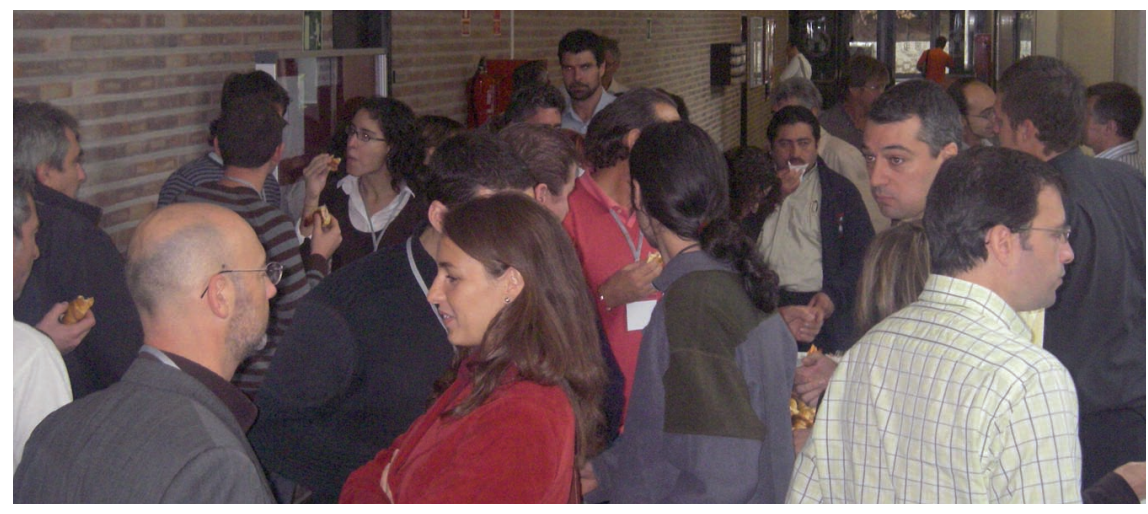

esta base de datos como referencia de calidad de las publicaciones españolas en sus procesos de evaluación de profesorado. Se complementa bien con los datos ofrecidos en la herramienta RESH (Revistas españolas de ciencias sociales y humanas) que aporta, especialmente, datos relativos al uso e influencia de las revistas, a partir de la elaboración de índices de citas que permiten calcular el impacto de cada una en el entorno disciplinar más próximo. No obstante, estas bases de datos deberían ser más sensibles a la inclusión de revistas procedentes de campos transversales y jóvenes como el de las CCAFD, ya que poseen pocas publicaciones periódicas de este campo.

Por su parte, Julio Martínez, bibliotecario de la Universidad de Las Palmas de Gran Canaria, analizó las principales deficiencias que aquejan a las publicaciones periódicas de las CCAFD. Entre ellas cabe destacar la falta de inversiones y de una política de promoción editorial adecuada, la falta de interés por las actividades de marketing y comercialización, edición y distribución deficientes, irregularidad en las tiradas, escasa visibilidad, existencia poco habitual del sistema de selección por expertos (peer review), y pobre profesionalización del proceso de edición. Desde su punto de vista, debería establecerse un periodo para adaptar la actual situación de las revistas de CCAFD a una nueva estrategia de edición en la que primara la calidad (p.e. cumpliendo con los criterios Latindex), concienciando a los organismos evaluadores de la actividad científica (Cneai, Aneca) de la necesidad de potenciar herramientas bibliométricas del campo que complementaran al Journal citation reports.

Las Jornadas finalizaron con la intervención de Kenneth Green, editor de la revista European physical education review, que presentó una serie de consideraciones alrededor del proceso de revisión por expertos basadas en su experiencia. Comenzó señalando que el proceso de revisión de un artículo no es infalible, ya que a veces se rechazan buenos artículos y otras se aceptan trabajos mucho peores. Entre los aspectos que deben tener en cuenta los revisores destacó, especialmente, la contribución que los artículos hagan al avance del conocimiento y el uso adecuado de la metodología. En este último caso sugirió contar siempre con un revisor o revisora especialista en la metodología empleada en el trabajo enviado a evaluación. Este profesor de la Universidad de Chester (Reino Unido) acabó con una serie de estrategias para conseguir publicar en revistas de calidad, tales como: sugerir sutilmente al editor algunos revisores, enviar un texto con buena escritura y presentación, responder a todos los comentarios de los revisores e incluir halagos por sus sugerencias, ser diplomático con sus preguntas, indicar la disponibilidad a nuevas aclaraciones, no ignorar las instrucciones para los autores y no rechazar ser revisor de una revista. 


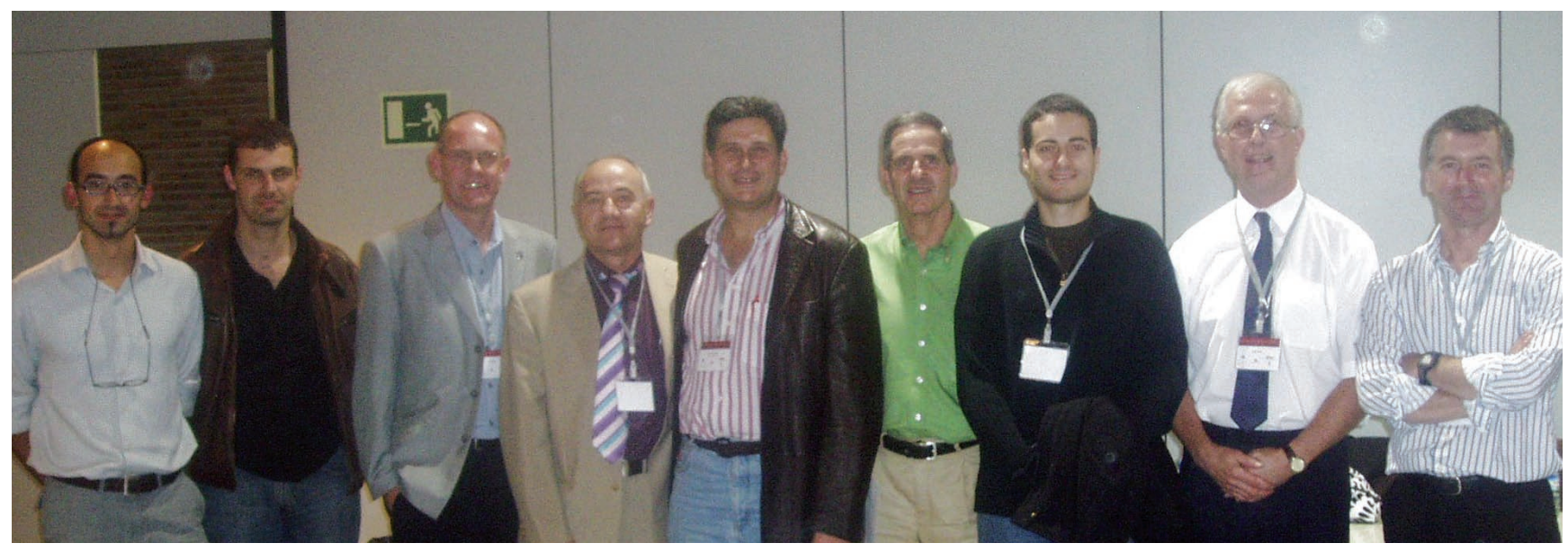

Javier Valenciano, David Brown, David Kirk, Tomàs Baiget, José Devís, Miguel Villamón, Vicente Beltrán, Alan Nevill y Ken Green.

\section{Conclusiones}

- Más allá del análisis de citas, la calidad de las revistas descansa en sus contenidos y en el rigor metodológico de los artículos. En este sentido, conviene incluir un experto en metodología entre los encargados de evaluar un manuscrito.

- La calidad también depende de la reputación de los editores y el buen hacer de los revisores. Se recomiensa la profesionalización de los procesos de gestión y edición de las revistas.

- La calidad de las revistas aumenta con su normalización, de manera que cumplan todos los criterios Latindex.

- El FI es solamente un indicador, ni el único ni el más apropiado en todos los casos. Existen muchos criterios, indicadores y modelos de evaluación de la calidad de las revistas que coinciden en sus aspectos fundamentales. De hecho, la emisión de juicios exige considerar múltiples fuentes de análisis o indicadores. Por otra parte, el concepto de "impacto" debería extenderse a la repercusión que un trabajo científico tiene a nivel social y personal.

- La calidad se incrementa con la visibilidad, y para ello la digitalización y el acceso libre en internet son estrategias clave.
- Los editores responsables de las revistas deberían impulsar encuentros de este tipo para contribuir a la mejora progresiva de sus publicaciones.

- La Fecyt, institución dedicada al apoyo de la investigación, debería impulsar iniciativas para mejorar la calidad de las revistas de CCAFD, como podrían ser evaluaciones especialmente dirigidas a este área. Igualmente, las instituciones con competencias en la misma, como el Consejo Superior de Deportes, deberían implicarse y respaldar dichas revistas, así como los estudios encaminados a mejorarlas.

Resaltamos que durante esos dos días de octubre se estableció una gran complicidad entre los asistentes y los ponentes y se observó una gran interacción entre ellos en forma de preguntas y comentarios. Las Jornadas cumplieron, por tanto, el objetivo de convertirse en un foro de intercambio y discusión de ideas y experiencias. No obstante, la meta última consistente en mejorar la calidad y el prestigio de las revistas españolas de CCAFD queda para más allá del encuentro, a la espera de la implicación de todas las personas participantes y las instituciones a las que representan para que finalmente se convierta en una realidad.

\section{Bibliografía}

Devís, José; Antolín, Luis; Villamón, Miguel; Moreno, Alberto; Valenciano, Javier. "Las revistas científico-técnicas españolas de las ciencias de la actividad física y el deporte: inventario y análisis de la calidad de contenido y difusión". En: Revista española de documentación científica, 2003, v. 26, n. 2, pp. 177-190.

Devís, José; Villamón, Miguel; Antolín, Luis; Valenciano, Javier; Moreno, Alberto. "Las revistas científico-técnicas españolas de ciencias de la actividad física y el deporte: adecuación a las normas ISO y grado de normalización". En: Ciência da informação, 2004, v. 33, n. 1, pp. 3847.

Villamón, Miguel; Devís, José; Valenciano, Javier. "Análisis de la visibilidad de las revistas científico-técnicas españolas de ciencias de la actividad física y el deporte". En: Revista de psicología del deporte, 2005, v. 14, n. 2, pp. 253-267.

Villamón, Miguel; Devís, José; Valenciano, Javier. "Análisis de las 'Instrucciones para autores' de las revistas españolas de ciencias de la actividad física y el deporte". En: Motricidad, 2006, n. 16, pp. 133-150.

Villamón-Herrera, Miguel; Devís-Devís, José; Valencia-Peris, Alexandra; Valenciano-Valcárcel, Javier. "Características y difusión de las revistas españolas de ciencias de la actividad física y el deporte". En: El profesional de la información, 2007, noviembre-diciembre, v. 16, n. 6, pp.????

Valenciano, Javier; Devís, José; Villamón, Miguel. "Análisis comparativo de la calidad de las revistas científico-técnicas españolas de ciencias de la actividad física y el deporte (2000-2005)". En: Information research: an international and electronic journal (en prensa).

José Devís-Devís; Miguel Villamón-Herrera, Departamento de Educación Física y Deportiva, Universitat de València.

C/ Gascó Oliag, 3. 46010 Valencia jose.devis@uv.es

miguel.villamon@uv.es 